40th "Jaszowiec" International School and Conference on the Physics of Semiconductors, Krynica-Zdrój 2011

\title{
Influence of Chemical Composition of Liquid Phase and Growth Process on Physical Properties of $\mathrm{Bi}_{2} \mathrm{Se}_{3}$, $\mathrm{Bi}_{2} \mathrm{Te}_{3}$ and $\mathrm{Bi}_{2} \mathrm{Te}_{2}$ Se Compounds
}

\author{
A. Hruban, A. Materna, W. Dalecki, G. Strzelecka, M. Piersa, \\ E. Jurkiewicz-Wegner, R. Diduszko, M. Romaniec and W. Oreowski \\ Institute of Electronic Materials Technology, Wólczyńska 133, 01-919 Warsaw, Poland \\ We studied synthesis and crystal growth of $\mathrm{Bi}_{2} \mathrm{Te}_{3}, \mathrm{Bi}_{2} \mathrm{Se}_{3}$ and $\mathrm{Bi}_{2} \mathrm{Te}_{2} \mathrm{Se}$ compounds by means of vertical \\ Bridgman method. Crystals were grown from stoichiometric melts and under different molar ratio of Bi:Te, Bi:Se \\ or Bi:Te:Se. The obtained crystals were characterized by X-ray diffraction analysis, energy dispersive X-ray \\ spectroscopy, scanning electron microscopy, atomic force microscopy, and the Hall effect measurements. Some of \\ the samples demonstrated insulating bulk behavior, by means of resistivity versus temperature measurements.
}

PACS: 71.55.-i, 72.10.-d, 72.15.-v, 72.20.-I, 73.20.-r

\section{Introduction}

$\mathrm{Bi}_{2} \mathrm{X}_{3}(\mathrm{X}=\mathrm{Te}, \mathrm{Se})$ and $\mathrm{Sb}_{2} \mathrm{Te}_{3}$ compounds are narrow gap semiconductors, belonging to a class of 3D topological insulators (TI), intensively investigated recently. Physical properties of $\mathrm{Bi}_{2} \mathrm{Te}_{3}, \mathrm{Bi}_{2} \mathrm{Se}_{3}$ and $\mathrm{Bi}_{2} \mathrm{Te}_{2}$ Se crystals are determined by layered structure and defect character of bulk electrical conductivity $[1,2,5]$.

Crystal structure of $\mathrm{Bi}_{2} \mathrm{X}_{3}$ consists of five-layer units of $\mathrm{Bi}$ and $\mathrm{X}$ hexagonal planes (quintuple), stacked along the $c$-axis with atomic order $\mathrm{X}-\mathrm{Bi}-\mathrm{X}-\mathrm{Bi}-\mathrm{X}$ (Fig. 1), or $\mathrm{Te}-\mathrm{Bi}-\mathrm{Se}-\mathrm{Bi}-\mathrm{Te}$ (Fig. 2), and form rhombohedral lattices in which the unit cell is composed of three quintuple layers. The units are stacked on the top of each other and are weakly bonded by the Van der Waals forces.

Real $\mathrm{Bi}_{2} \mathrm{X}_{3}$ crystals tend to contain high concentration of point defects i.e. bismuth antisite $\mathrm{Bi}_{\mathrm{Te}}$ (acceptor) in $\mathrm{Bi}_{2} \mathrm{Te}_{3}$ and selenium vacancies $\mathrm{V}_{\mathrm{Se}}$ (donor) in $\mathrm{Bi}_{2} \mathrm{Se}_{3}$.

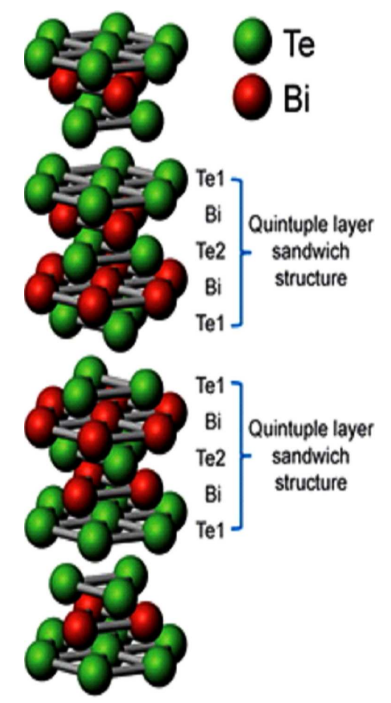

Fig. 1. Layered crystal structure of $\mathrm{Bi}_{2} \mathrm{Te}_{3}$ [3].

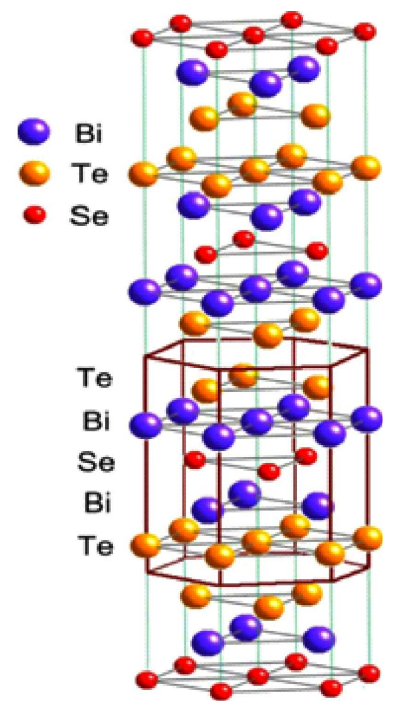

Fig. 2. Layered crystal structure of $\mathrm{Bi}_{2} \mathrm{Te}_{2} \mathrm{Se}$ crystal [4].

As a result, undoped stoichiometric $\mathrm{Bi}_{2} \mathrm{Te}_{3}$ is of $p$-type conductivity with a typical carrier concentration $p=$ $(1-5) \times 10^{19} \mathrm{~cm}^{-3}$, and $\mathrm{Bi}_{2} \mathrm{Se}_{3}$ shows $n$-type conductivity with $n=(1-10) \times 10^{19} \mathrm{~cm}^{-3}$. Both of them present highly metallic behavior. In order to see topological surface contribution to electron transport, it is necessary to grow crystals with decreased bulk carrier concentration. It can be realized by compensating doping, or suppressing of point defects concentration.

\section{Experiment}

In this work we investigated the influence of chemical composition of liquid phase and thermal conditions of the growth process on physical properties of $\mathrm{Bi}_{2} \mathrm{Se}_{3}, \mathrm{Bi}_{2} \mathrm{Te}_{3}$ and $\mathrm{Bi}_{2} \mathrm{Te}_{2}$ Se compounds. 
Synthesis and crystallization were performed in home-made vertical Bridgman setup with six heating zones, allowing for control and modification of temperature profiles, necessary for obtaining crystals of each compound. Molar ratio of the elements used for synthesis and crystal growth in the consecutive processes was changed from stoichiometric to anion rich in the following range:

$$
\begin{aligned}
& \mathrm{Bi}_{2} \mathrm{Te}_{3} \rightarrow \mathrm{Bi}_{1.82} \mathrm{Te}_{3.18}, \quad \mathrm{Bi}_{2} \mathrm{Se}_{3} \rightarrow \mathrm{Bi}_{1.75} \mathrm{Se}_{3.25}, \\
& \mathrm{Bi}_{2} \mathrm{Te}_{2} \mathrm{Se} \rightarrow \mathrm{Bi}_{1.97} \mathrm{Te}_{2.19} \mathrm{Se}_{0.84} .
\end{aligned}
$$

High purity $(5-6 \mathrm{~N})$ elements were charged into a quartz ampoule, sealed under vacuum $10^{-5} \mathrm{Tr}$ and then placed in a vertical furnace for both synthesis and crystal growth. The ampoule with molten charge of 100-150 g was lowered in the furnace with a constant rate of 1$3 \mathrm{~mm} / \mathrm{h}$.

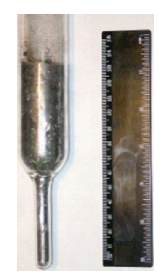

Fig. 3. $\mathrm{Bi}_{2} \mathrm{Te}_{3}$ crystal grown by vertical Bridgman method.

After solidification crystals were annealed for $48 \mathrm{~h}$ in aim of homogenization and structure ordering. One of the crystals grown by this technique is shown in Fig. 3.

The obtained crystals were characterized by the following methods:

- X-ray diffractometry (XRD),

- energy dispersive X-ray Spectroscopy (EDX),

- scanning electron microscopy (SEM),

- atomic force microscopy (AFM),

- the Hall and conductivity measurements.

Samples for investigations were prepared by exfoliation technique.

\section{Results}

Powder X-ray diffractometer Siemens D-500 was used for structural analysis of the crystals. Figure 4 shows the diffractograms of $\mathrm{Bi}_{2} \mathrm{Te}_{3}$ and $\mathrm{Bi}_{2} \mathrm{Te}_{2} \mathrm{Se}$. They confirm existence of single phase crystals.

EDX spectroscopy with use of scanning microscope (SEM) AURIGA (ZEISS) was applied for chemical analysis of crystal composition. Results of the analysis are presented in Table together with the atomic ratio of chemical composition as charged into the ampoule. These results prove that the chemical composition in the crystal is well maintained.

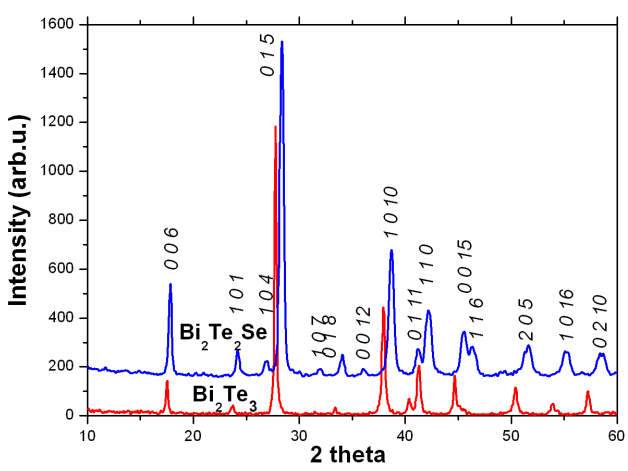

Fig. 4. X-ray diffractogram of $\mathrm{Bi}_{2} \mathrm{Te}_{2} \mathrm{Se}$ and $\mathrm{Bi}_{2} \mathrm{Te}_{3}$.

Chemical composition.

TABLE

\begin{tabular}{c|c|c|c}
\hline \hline Compound & Element & $\begin{array}{c}\text { Growth as mixed } \\
\text { [at.\%] }\end{array}$ & $\begin{array}{c}\text { EDX } \\
\text { [at.\%] }\end{array}$ \\
\hline \multirow{2}{*}{$\mathrm{Bi}_{2} \mathrm{Se}_{3}$} & $\mathrm{Bi}$ & 40 & 38.64 \\
& $\mathrm{Se}$ & 60 & 61.36 \\
\hline $\mathrm{Bi}_{2} \mathrm{Te}_{3}$ & $\mathrm{Bi}$ & 40 & 38.69 \\
& $\mathrm{Te}$ & 60 & 61.31 \\
\hline \multirow{2}{*}{$\mathrm{Bi}_{2} \mathrm{Te}_{1.95} \mathrm{Se}_{1.05}$} & $\mathrm{Bi}$ & 40 & 39.27 \\
& $\mathrm{Te}$ & 38 & 38.18 \\
& $\mathrm{Se}$ & 22 & 22.54
\end{tabular}

The layered structure of the studied material (of samples prepared by exfoliation technique) was nicely visible by means of SEM and AFM techniques. In Fig. 5 cross-section of some blocks of $\mathrm{Bi}_{2} \mathrm{Se}_{3}$ (BS 13) crystal is presented.

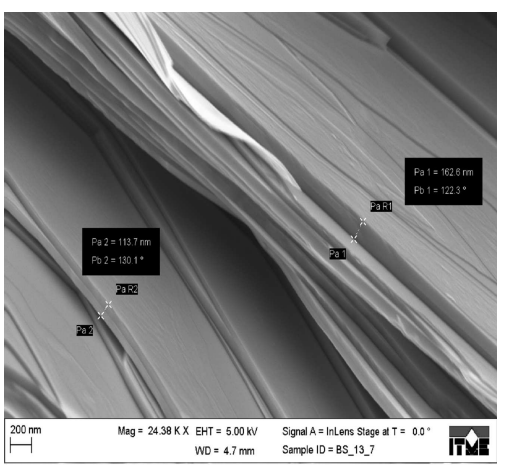

Fig. 5. Blocks of quintuples with thickness of $113.7 \mathrm{~nm}$ and $162.6 \mathrm{~nm}$.

AFM technique allows to observe single quintuple layer on the cleavage crystal surface (Fig. 6). One quintuple thickness is about $1 \mathrm{~nm}$. It is according to data presented by Kim et al. [7] and Hong et al. [8].

The Hall effect measurements were performed for van der Pauw configuration, in temperature range 6-300 K and under low magnetic field regime. The measured sam- 


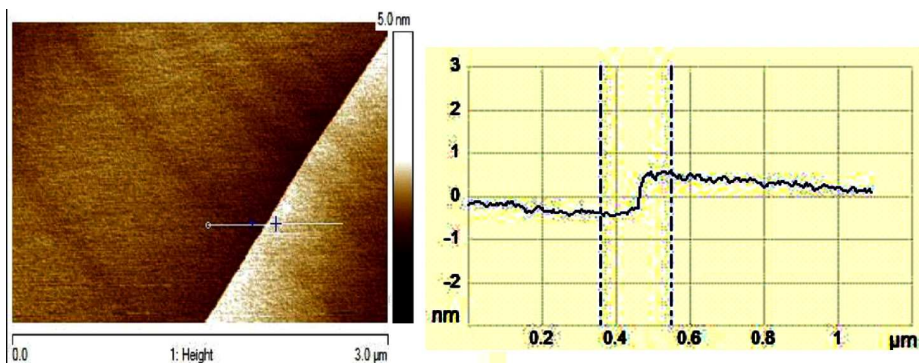

Fig. 6. AFM image of $\mathrm{Bi}_{2} \mathrm{Se}_{3}$ surface. a) Single quintuple layer, b) the height profile along line in a) showing $1 \mathrm{~nm}$ layer.

ples were cut from different regions of the ingot. Segregation effects allowed to monitor crystal properties dependent on defect concentration in a convenient way. This regarded not only electric transport parameters but also all other parameters investigated.

\section{1. $B i_{2} T e_{3}$}

In Fig. 7 distribution of carrier concentration along $\mathrm{Bi}_{2} \mathrm{Te}_{3}$ ingots for crystal grown from the melt with excess of Te is shown. Increasing Te excess causes carrier concentration decreasing from $1.5 \times 10^{19} \mathrm{~cm}^{-3}$, down to $4 \times 10^{18} \mathrm{~cm}^{-3}$ in $p$-type part of the ingot $\mathrm{Bi}_{2} \mathrm{Te}_{3}$ (no. BT 6 ). In the end part of these crystals, conductivity type changes from " $p$ "-type to " $n$ "-type. In the process with highest excess of Te in the melt, crystal of $n$-type conductivity in whole volume was obtained (no. BT 9). It is connected with strong decrease of antisite defect $\mathrm{Bi}_{\mathrm{Te}}$ acceptor and/or increase of unknown donor defect.

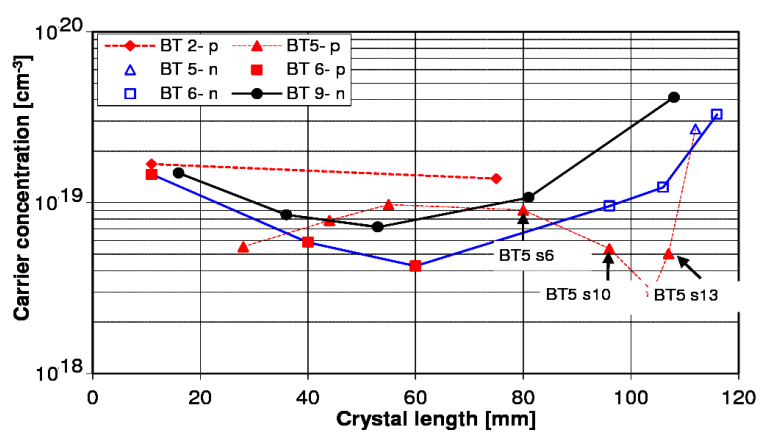

Fig. 7. Suppressing of point defects $\left(\mathrm{Bi}_{\mathrm{Te}}\right)$ concentration in $\mathrm{Bi}_{2} \mathrm{Te}_{3}$ crystals grown from the stoichiometric melt (no. BT2) with 4\% (no. BT5), 8\% (no. BT6), and $15 \%$ (no. BT9) excess of Te.

Low temperature resistivity measurements were performed on the $p$-type samples cut from different parts of $\mathrm{Bi}_{2} \mathrm{Te}_{3}$ crystal to find out the material which exhibits insulating bulk state. Results are presented in Fig. 8. Resistivity plot vs. temperature of BT-5s6 and BT-5s10 samples (explanation of sample names is provided in

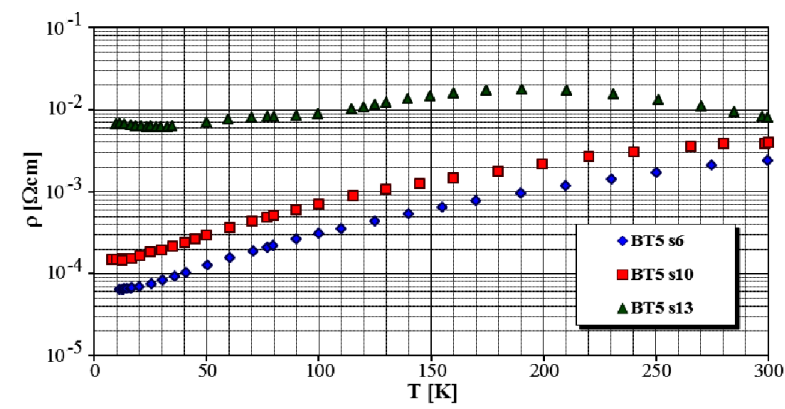

Fig. 8. Resistivity dependence on temperature of $\mathrm{Bi}_{2} \mathrm{Te}_{3}$ crystal.

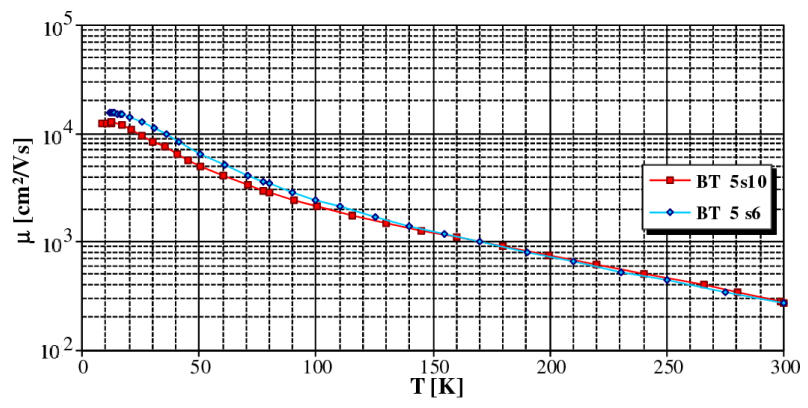

Fig. 9. Mobility dependence on temperature of $\mathrm{Bi}_{2} \mathrm{Te}_{3}$ crystal.

Fig. 7) show resistivity with temperature increase characteristic for metallic behavior. On the other hand, the plot for the sample BT-5s 13 is similar to the one presented in paper [5], and it is characteristic for insulating bulk behavior. For samples BT5s6 and s10 carrier mobility at temperature $10 \mathrm{~K}$ (Fig. 9) was $\mu=1.5 \times 10^{4} \mathrm{~cm}^{2} \mathrm{~V}^{-1} \mathrm{~s}^{-1}$.

\section{2. $B i_{2} \mathrm{Se}_{3}$}

The effect of suppressing of electron (resulted from $\mathrm{V}_{\mathrm{Se}}$ defects)[6] concentration in $\mathrm{Bi}_{2} \mathrm{Se}_{3}$ crystals with $\mathrm{Se}$ excess is presented in Fig. 10. Compared to stoichiometric

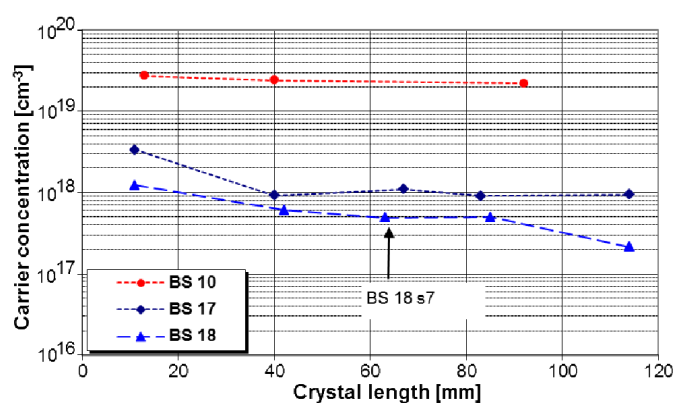

Fig. 10. Influence of melt composition on suppressing of carrier concentration $(n) \mathrm{Bi}_{2} \mathrm{Se}_{3}$ crystals: BS-10 stoichiometric crystal, BS-17 [ $\left[\mathrm{Bi}_{1.75} \mathrm{Se}_{3.25}\right]$, and BS-18 $\left[\mathrm{Bi}_{1.72} \mathrm{Se}_{3.30}\right]$ - Se excess. 


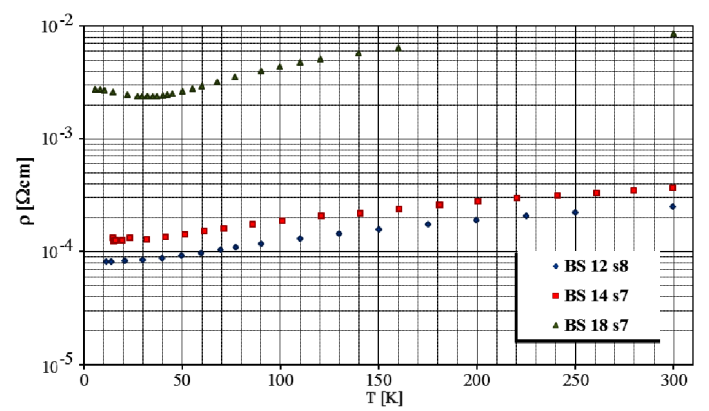

Fig. 11. Resistivity versus temperature dependence of $\mathrm{Bi}_{2} \mathrm{Se}_{3}$ crystal.

material, the concentration decreases by almost two orders of magnitude for $\mathrm{Bi}_{1.7} \mathrm{Se}_{3.3}$ sample (no. $\mathrm{BS} 18$ ). In Fig. 11 plot of resistivity versus temperature are shown for the same crystal as in Fig. 10. The resistivity of the crystal grown from stoichiometric melt presents typical metallic behavior. In material with decreased carrier concentration to the level $(2-5) \times 10^{17} \mathrm{~cm}^{-3}$ small decrease of resistivity is observed in the temperature range from the room temperature down to $50 \mathrm{~K}$. However, below $50 \mathrm{~K}$ the resistivity changes are typical for semiconducting material. Such crystals give a chance to obtain material with $p$-type bulk of low level of acceptor concentration in which topological effects of metallic surface could be observed.

\section{3. $\mathrm{Bi}_{2} \mathrm{Te}_{2} \mathrm{Se}$}

Experiments of synthesis and crystal growth of $p$-type material with low carrier concentration were performed for ternary compound from stoichiometric $\mathrm{Bi}_{2} \mathrm{Te}_{2} \mathrm{Se}$ to $\mathrm{Bi}_{2.00} \mathrm{Te}_{2.20} \mathrm{Se}_{0.80}$. In Fig. 12 distribution of carrier concentration along ternary crystals is shown. Crystal no. BTS $1\left(\mathrm{Bi}_{2.00} \mathrm{Te}_{1.95} \mathrm{Se}_{1.05}\right)$ shows $p$-type conductivity only, no. BTS $5\left(\mathrm{Bi}_{2.00} \mathrm{Te}_{2.05} \mathrm{Se}_{0.95}\right)$ changes from $p$-type to $n$-type in the ingot end. Crystals no. BTS 7, 8, 9 (composition from $\mathrm{Bi}_{2.00} \mathrm{Te}_{2.12} \mathrm{Se}_{0.88}$ to $\left.\mathrm{Bi}_{2.00} \mathrm{Te}_{2.20} \mathrm{Se}_{0.80}\right)$ show $n$-type conductivity.

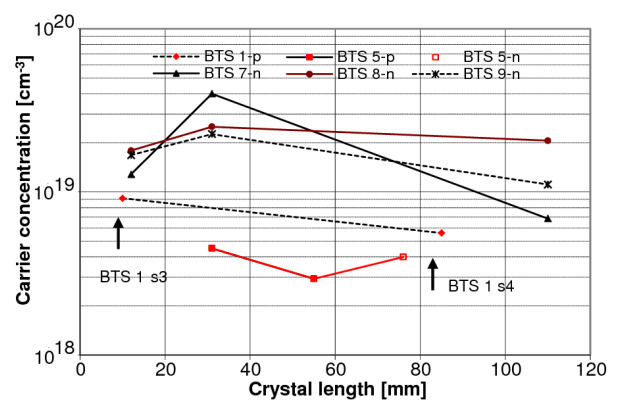

Fig. 12. Carrier concentration distribution along ternary $\mathrm{Bi}_{2} \mathrm{Te}_{2} \mathrm{Se}$ crystals with different composition: no. BTS $1-\mathrm{Bi}_{2.00} \mathrm{Te}_{1.95} \mathrm{Se}_{1.05}$, no. BTS $5-$ $\mathrm{Bi}_{2.00} \mathrm{Te}_{2.05} \mathrm{Se}_{0,95}$ crystals, no. BTS 7, 8, 9 - composition from $\mathrm{Bi}_{2.00} \mathrm{Te}_{2.12} \mathrm{Se}_{0.88}$ to $\mathrm{Bi}_{2.00} \mathrm{Te}_{2.20} \mathrm{Se}_{0.80}$.

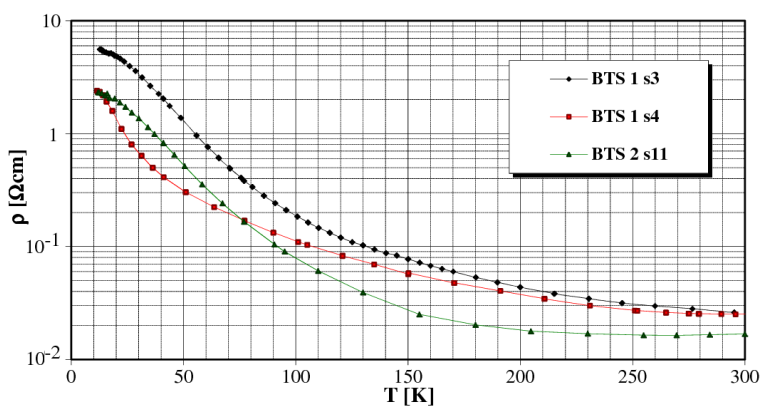

Fig. 13. Resistivity $(\rho)$ vs. temperature dependence of $\mathrm{Bi}_{2.00} \mathrm{Te}_{1.95} \mathrm{Se}_{1.05}$ crystals.

In Fig. 13 resistivity dependence on temperature is shown. High resistivity increase, of about two orders of magnitude, is observed for temperature decreasing from 300 down to $10 \mathrm{~K}$. In the temperature range below $50 \mathrm{~K}$ the change of conductivity type from $p$ to $n$ occurs. It means the change of dominant charge carriers from holes to electrons. According to [4], ternary compound $\mathrm{Bi}_{2} \mathrm{Te}_{2} \mathrm{Se}$ is the best material to observe topological insulator behavior.

\section{Conclusions}

In this work we studied crystal growth of $\mathrm{Bi}_{2} \mathrm{Te}_{3}$, $\mathrm{Bi}_{2} \mathrm{Se}_{3}, \mathrm{Bi}_{2} \mathrm{Te}_{2} \mathrm{Se}$ compounds. Undoped crystals with controlled stoichiometry by chemical composition of the liquid phase were obtained. In some crystals insulating bulk behavior was observed. However, the highest increase of resistivity with temperature decrease were observed for $\mathrm{Bi}_{2} \mathrm{Te}_{2}$ Se crystals.

\section{References}

[1] Y. Xia, D. Qian, D. Hsieh, L. Wray, A. Pal, H. Lin, A. Bansil, D. Grauer, Y.S. Hor, R.J. Cava, M.Z. Hasan, Nature Phys. 5, 398 (2009).

[2] H. Zhang, C.-X. Liu, X.-L. Qi, X. Dai, Z. Fang, S.C. Zhang, Nature Phys.5, 438 (2009).

[3] M.Z. Hasan, C.L. Kane, arXiv:1001.3895v1, Feb 2010.

[4] W. Zhang, R. Yu, H.-J. Zhang, X. Dai, Z. Fang, New J. Phys. 12, (2010).

[5] Zhi Ren, A.A. Taskin, S. Sasaki, K. Segawa, Y. Ando, Phys. Rev. B 82, 241 (2010).

[6] Y.S. Hor, A. Richardella, M.Z. Hasan, N.P. Ong, R.J. Cava, Phys. Rev. B 79, 195 (2009).

[7] Y.S. Kim, M. Brahlek, N. Bansal, E. Edrey, G.A. Kapilevich, K. Iida, M. Tamimura, Y. Horibe, S.-W. Cheong, S. Oh, Condensed Matter, arXiv:1104.0913.

[8] S.S. Hong, W. Kundhikanjana, J.J. Cha, K. Lai, D. Kong, S. Meister, M.A. Kelly, Z. Xun, Nano Lett. 10, 3118 (2010); DOI:10102/nL101884. 